Revista Brasileira de

Engenharia Agrícola e Ambiental

v.13, n.4, p.470-476, 2009

Campina Grande, PB, UAEA/UFCG - http://www.agriambi.com.br

Protocolo 190.07 - 03/12/2007 • Aprovado em 21/11/2008

agriambi

\title{
Umidade de equilíbrio de café cereja descascado baseada em métodos estático e dinâmico
}

\author{
José D. Henao ${ }^{1}$, Marlene R. de Queiroz ${ }^{2} \&$ Niurka M.A. Haj-Isa ${ }^{3}$
}

\begin{abstract}
RESUMO
Construíram-se as isotermas de dessorção de grãos da espécie Café Arábica (Coffea arábica L.), da cultivar Novo Mundo-Acaiá, para as temperaturas de 50 e $73{ }^{\circ} \mathrm{C}$ e teores de mucilagem residual de 16,1 e $52,7 \%$; posteriormente, oito modelos matemáticos de sorção de produtos higroscópicos foram ajustados aos dados experimentais (BET, BET Linear, GAB, Halsey, Halsey Modificado, Langmuir, 0 swin e Peleg), com a finalidade de se obter seus coeficientes. A escolha do melhor ajuste se deu em função da avaliação do coeficiente de determinação $\left(R^{2}\right)$, do desvio relativo médio (DRM), da tendência de distribuição dos resíduos provenientes dos ajustes dos modelos e do desvio padrão da estimativa. As isotermas de dessorção para temperatura de $50^{\circ} \mathrm{C}$ e teores de mucilagem residual de 16,1 e $52,7 \%$ podem ser representadas pelos modelos Peleg, Halsey Modificado, $\mathrm{GAB}$ e $\mathrm{O}$ swin. Para a temperatura de $73^{\circ} \mathrm{C}$, o modelo $\mathrm{GAB}$ não representa bem os dados experimentais, sendo que os modelos Peleg e Halsey Modificado indicam o melhor desempenho. Com vistas à estimativa das umidades de equilíbrio dinâmicas, a equação proposta por Fioreze mostrou-se altamente satisfatória.
\end{abstract}

Palavras-chave: Coffea arábica L, isotermas de sorção, teor de mucilagem

\section{Equilibrium moisture contents for peeled coffee cherry based on static and dynamic methods}

\begin{abstract}
The desorption isotherms for 50 and $73^{\circ} \mathrm{C}$ and residual mucilage content of 16.1 and $52.7 \%$ in Arabica Coffee (Coffea arábica L.), cv N ovo M undo-A caiá, were built. After this, eight mathematical models (BET, BET Linear, GAB, Halsey, Halsey Modified, Langmuir, O swin e Peleg) for desorption for hygroscopic products were adjusted to the experimental data for obtaining the coefficients of the models. The choice of the best adjustment was based on the analysis of the following parameters: determination coefficient $\left(R^{2}\right)$, relative average deviation, residual distribution tendency and standard deviation of the estimation. The desorption isotherms at $50 \stackrel{\circ}{\mathrm{C}}$ for cherry coffee with 16.1 and $52.7 \%$ residual mucilage contents could be represented by Peleg, Modified Halsey, GAB e 0 swin models. $A t 73^{\circ} \mathrm{C}$ the $\mathrm{GAB}$ model did not represent the experimental data, and the models of Peleg and Modified $\mathrm{H}$ alsey were the best ones. In order to estimate the dynamic equilibrium moisture content, the equation proposed by Fioreze showed to be highly satisfactory.
\end{abstract}

Key words: Coffea arábica L, sorption isotherms, mucilage content

\footnotetext{
1 Universidad Surcolombiana, Avenida Pastrana, Carrera primera. Facultad de Ingenieria, Neiva, Huila, Colômbia. Fone (57-8-8758775).E-mail: duvanhena023@hotmail.com

2 FEA GRI/U NICAM P, CP 6011, Cidade U niversitária Zeferino Vaz s/n. CEP 13083-875, Campinas, SP. Fone (19) 3521 1073, Fax (19) 3521 1010. E-mail: marlene@agr.unicamp.br

${ }^{3}$ FAJ/FAM/CEU NSP, Rod. Adhemar de Barros - Km 127 - Pista Sul - Jaguariúna/SP - CEP 13.820-000. Jaguariúna, SP. Fone (19) 38378500. E-mail: professoraniurka@hotmail.com
} 


\section{INTRODUÇÃO}

Segundo Fortes \& Okos (1978), uma isoterma é simplesmente a curva que relaciona o conteúdo de umidade de equilíbrio de um produto com a umidade relativa do ar. O conhecimento sobre os fenômenos de sorção de umidade é fundamental para se abordar e entender o processo de secagem.

De acordo com Daudin (1983), as isotermas de sorção, freqüentemente chamadas curvas de sorção, traduzem a higroscopicidade do produto, definida pelas relações existentes entre a água e os outros componentes. Segundo o autor, em plano teórico, graças a essas curvas e a partir dos valores do teor de água e da temperatura da superfície do produto, é possível conhecer o valor da pressão parcial de vapor de água na superfície e, por conseguinte, calcular a taxa de secagem.

A atividade de água $\left(\mathrm{a}_{\mathrm{w}}\right)$, conhecida também como umidade relativa de equilíbrio, foi definida por Scott (1957) e Salwin \& Slawson (1959), como a relação entre a pressão de vapor de água no ar e a pressão de vapor de água no ar saturado, medidas a mesma temperatura.

Pode-se entender a atividade de água como a medida de água livre disponível em um alimento, isto é, que não está vinculada a outros componentes e, portanto, pode reagir quimicamente, permitindo ainda o crescimento de microrganismos. Labuza et al. (1985) acrescentaram que a $a_{w}$ afeta a estabilidade de alimentos desidratados controlando diretamente tanto a taxa de atividade microbiana como as reações químicas, e pode ser aumentada, seja por ganho de umidade da atmosfera ou por incremento da temperatura do produto.

A equação matemática da isoterma de sorção de umidade descreve a relação entre $\mathrm{a}_{\mathrm{w}}$ e o conteúdo de umidade de equilíbrio para um produto alimentício (Lomauro et al., 1985). As isotermas de sorção de umidade em pesquisas de alimentos são usadas para vários propósitos, como determinação do tempo de secagem ou, ainda, predições para misturar e embalar alimentos, prevendo as mudanças de umidade que ocorrem durante a armazenagem e, consequientemente, a sua estabilidade.

As curvas de sorção de umidade podem ser representadas, matematicamente, através de equações de isotermas de equilíbrio. Na literatura existem diversas equações empíricas, modelos matemáticos que são utilizados para representar as curvas experimentais de sorção em alimentos.

Ante o exposto se propôs, neste trabalho, a determinação das isotermas de dessorção de café cereja descascado com teores de mucilagem residual de 16,1 e 52,7\% para as temperaturas de 50 e $73{ }^{\circ} \mathrm{C}$ e o posterior ajuste de modelos matemáticos. De posse desses resultados, pretende-se estimar as umidades de equilíbrio dinâmico.

\section{MATERIAL E MÉTODOS}

Utilizaram-se grãos da espécie Café Arábica (Coffea arábica L.), da cultivar Novo Mundo-Acaiá, da fazenda Vargem Grande, localizada no município de Jacutinga, MG, a $1200 \mathrm{~m}$ de altitude, colhidos em arbustos de cinco anos de idade.
A colheita foi realizada na safra de 2004. O método de colheita adotado foi a derriça manual no pano, privilegiandose os grãos totalmente maduros.

O café foi colhido nas primeiras horas da manhã e levado para o Laboratório de Tecnologia Pós-colheita/Secagem, da Faculdade de Engenharia Agrícola da UNICAMP, onde se procederam a lavagem e a separação por densidade dos grãos sobremaduros, denominados bóias; posteriormente, foram separados os grãos verdes, mediante inspeção visual e pressão dos dedos. A lavagem do café foi realizada em um tanque plástico e os grãos sobremaduros ou bóias foram separados por flutuação, removidos posteriormente com a ajuda de uma peneira. Por outro lado, o descascamento do café cereja foi realizado mediante o uso de um despolpador mecânico, tipo horizontal, marca Pinhalense, Modelo DPM-02, ano 2003. As cascas remanescentes foram eliminadas manualmente. Este equipamento era usado para a produção de grãos apenas descascados.

\section{Determinação de mucilagem total}

Utilizou-se um quilograma de café cereja apenas descascado, denominado amostra total (AT); a desmucilagem total foi realizada colocando-se o material em recipiente plástico contendo $400 \mathrm{~g}$ de água e se atritando os grãos entre si, por agitação, com a ajuda de uma batedeira marca Britania - Pérola/Plus operada em potência máxima, cuja haste do agitador foi protegida com fibra a fim de evitar danos mecânicos aos grãos; a água era renovada mantendo-se a proporção inicial e o material agitado, até que o atrito entre os grãos produzisse ruído característico de lixa; em seguida, drenou-se a massa e se pesou o café resultante, denominado amostra totalmente desmucilada (AD). Com esses dados, calculou-se o teor de mucilagem (TM), de acordo com a Eq. 1.

$$
\mathrm{TM}=(\mathrm{AT}-\mathrm{AD}) / \mathrm{AD}
$$

A desmucilagem parcial das amostras seguiu procedimento semelhante, utilizando-se diferentes proporções água/matéria-prima e distintos tempos de agitação.

O teor de mucilagem residual (TM') foi calculado conforme a Eq. 2.

$$
\mathrm{TM}^{\prime}=\left(\mathrm{AD}^{\prime}-\mathrm{AD}\right) / \mathrm{AD}
$$

donde: AD' - Massa da amostra desmucilada parcialmente; AD - Massa da amostra desmucilada totalmente e TM'- Teor de mucilagem da $\mathrm{AD}^{\prime}$ ( $\mathrm{g}$ de mucilagem $\mathrm{g}^{-1}$ de $\mathrm{AD}$ ).

Considerando-se que, a medida em que a safra avança, há maior dificuldade em se retirar a mucilagem; esta metodologia foi complementada com a determinação dos sólidos totais presentes na mucilagem removida, através do método $\mathrm{n}^{\circ} 22.018$ para determinação de umidade de frutas e produtos de frutas (AOAC, 1995) e os sólidos totais foram calculados por diferença, desta maneira, a mucilagem residual pode ser representada pelo cálculo do percentual do total de sólidos presentes na mucilagem do café cereja descascado. 


\section{Umidade de equilíbrio - método estático}

Determinou-se a umidade de equilíbrio dos cafés cereja descascados com dois níveis de mucilagem residual, $\mathrm{M} 1=$ $16,1 \%$ e M2 $=52,7 \%$, a partir do método estático gravimétrico, utilizando-se soluções saturadas de sais para temperaturas de 50 e $73{ }^{\circ} \mathrm{C}$, respectivamente (Tabela 1); previamente, determinou-se o conteúdo de umidade das amostras.

Para a determinação da umidade de equilíbrio, amostras de cerca de $4 \mathrm{~g}$ de café cereja descascado, acondicionadas em cadinhos plásticos com 3 repetições, foram colocadas em recipientes herméticos contendo diferentes soluções saturadas de sais (Tabela 1). Cada conjunto de frascos foi colocado em estufas mantidas em temperaturas de 50 e $73{ }^{\circ} \mathrm{C}$, respectivamente, até que as amostras atingissem o equilíbrio.

$\mathrm{O}$ ponto de equilíbrio foi definido mediante pesagens sucessivas das amostras em balança analítica, marca Scientech, modelo SA 210 com precisão de $0,0001 \mathrm{~g}$, até que ocorresse a estabilização da massa das amostras na quarta casa decimal. Uma vez atingido o equilíbrio, as amostras foram levadas a uma estufa da marca FANEM, modelo 320-SE, para determinação da umidade, segundo a metodologia da AOAC (1995).

Analisaram-se as isotermas de sorção de acordo com os seguintes modelos matemáticos citados, entre outros, por Lomauro et al. (1985), Figueira et al. (2004), Toneli (2006): BET, BET Linear, GAB, Halsey, Halsey Modificado, Langmuir, Oswin e Peleg, correspondentes, respectivamente, às Eqs 3 a 10:

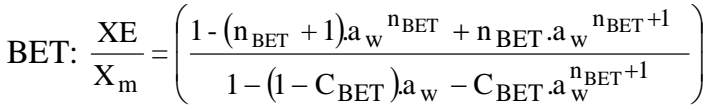

$$
\begin{aligned}
& \text { BET linear: } \frac{\mathrm{a}_{\mathrm{w}}}{\left(1-\mathrm{a}_{\mathrm{w}}\right) \mathrm{XE}}=\frac{1}{\mathrm{X}_{\mathrm{m}} \mathrm{C}_{\mathrm{BET}}}+\frac{\mathrm{a}_{\mathrm{w}}\left(\mathrm{C}_{\mathrm{BET}}-1\right)}{\mathrm{X}_{\mathrm{m}} \mathrm{C}_{\mathrm{BET}}} \\
& \text { GAB: } X E=\frac{X_{m} C_{G A B} K_{G A B} a_{w}}{\left(1-K_{G A B} a_{w}\right)\left(1-K_{G A B} a_{w}+C_{G A B} K_{G A B} a_{w}\right)} \\
& \text { Halsey: } a_{w}=\exp \left(-\mathrm{A}_{\mathrm{H}} / \mathrm{XE}^{\mathrm{b}_{\mathrm{H}}}\right) \\
& \begin{array}{c}
\text { Halsey } \\
\mathrm{a}_{\mathrm{w}}
\end{array} \text { Modificado }\left(\mathrm{X}_{\mathrm{m}}\right) \exp \left(-\mathrm{A}_{\mathrm{H}} / \mathrm{XE}^{\mathrm{b}_{\mathrm{H}}}\right) \\
& \text { Langmuir: } \frac{X E}{X_{m}}=\frac{C_{L} a_{w}}{1+C_{L} a_{w}} \\
& \text { Oswin: } X E=A_{O}\left(\frac{a_{w}}{1-a_{w}}\right)^{B_{o}} \\
& \text { Peleg: } X E=k_{1} a_{w}{ }^{n_{1}}+k_{2} a_{w}{ }^{{ }^{n}}
\end{aligned}
$$

donde:

$\mathrm{A}_{\mathrm{H}}, \mathrm{b}_{\mathrm{H}}$ - Constantes de ajuste do modelo de Halsey e Halsey Modificado

$\mathrm{A}_{\mathrm{O}}, \mathrm{B}_{\mathrm{O}}$ - Constantes de ajuste do modelo de Oswin Aw - Atividade de água
$\mathrm{C}_{\mathrm{BET}}$ - Constante de ajuste dos modelos de BET e BET linear

$\mathrm{C}_{\mathrm{GAB}}, \mathrm{K}_{\mathrm{GAB}}$ - Constante de ajuste do modelo de $\mathrm{GAB}$

$\mathrm{C}_{\mathrm{L}}$ - Constante de ajuste do modelo de Langmuir $\mathrm{n}_{1}, \mathrm{n}_{2}, \mathrm{k}_{1}, \mathrm{k}_{2}$ - Constantes de ajuste do modelo de Peleg

$\mathrm{n}_{\mathrm{BET}}$ - Constante de ajuste do modelo de BET

$\mathrm{XE}$ - Umidade de equilibrio $\left(\mathrm{kg}_{\mathrm{H} 2 \mathrm{O}} \mathrm{kg}_{\mathrm{Ms}}{ }^{-1}\right)$

$\mathrm{Xm}$ - Umidade na monocamada $\left(\mathrm{kg}_{\mathrm{H} 2 \mathrm{O}} \mathrm{kg}_{\mathrm{Ms}}{ }^{-1}\right)$

Realizou-se o ajuste dos modelos matemáticos aos dados experimentais através do pacote de estimativa não-linear (Statistica $6.0^{\circledR}$ ) e o critério utilizado para avaliação do ajuste foi o módulo do desvio relativo médio (Eq. 11):

$$
\mathrm{DRM}=\frac{100}{\mathrm{n}} \sum_{\mathrm{i}=1}^{\mathrm{n}} \frac{|\mathrm{VP}-\mathrm{VE}|}{\mathrm{VE}}
$$

donde:

DRM - Desvio relativo médio

n - Número de observações experimentais

VE - Valor experimental $\left(\mathrm{g}_{\text {água }} \mathrm{g}_{\mathrm{ms}}{ }^{-1}\right)$

VP - Valor predito pelo modelo ( $g_{\text {água }} \mathrm{g}_{\mathrm{ms}}{ }^{-1}$ )

Geralmente, considera-se que valores de DRM abaixo de $10 \%$ indicam ajuste adequado para aplicações práticas (Aguerre et al., 1985).

Além do desvio relativo médio, considerou-se a avaliação da tendência de distribuição dos resíduos provenientes dos ajustes dos modelos, calculados pela Eq. 12, e a avaliação do desvio padrão da estimativa (SE), calculado de acordo com a Eq. 13. O valor de SE é inversamente proporcional à qualidade do ajuste do modelo matemático.

$$
\begin{gathered}
e_{i}=V P-V E \\
S E=\sqrt{\frac{\sum_{i=1}^{n}(V P-V E)^{2}}{G L}}
\end{gathered}
$$

donde:

$$
\begin{aligned}
& \left.\mathrm{e}_{\mathrm{i}} \text { - Resíduo ( } \mathrm{g}_{\text {água }} \mathrm{g}_{\mathrm{ms}}{ }^{-1}\right) \\
& \text { G - Graus de liberdade do modelo }
\end{aligned}
$$

\section{Umidade de equilíbrio - método dinâmico}

\begin{tabular}{|c|c|c|c|c|c|c|c|c|c|}
\hline \multicolumn{2}{|c|}{ Sal } & $\mathrm{NaOH}$ & $\mathrm{KOH}$ & $\mathrm{LiCl}$ & KF & $\mathrm{K}_{2} \mathrm{CO}_{3}$ & $\mathrm{NaBr}$ & KI & $\mathrm{NaCl}$ \\
\hline \multirow{2}{*}{$a_{w}$} & $50^{\circ} \mathrm{C}$ & 0,0494 & 0,0572 & 0,111 & 0,208 & 0,427 & 0,5093 & 0,6449 & 0,7443 \\
\hline & $73^{\circ} \mathrm{OC}$ & 0,0225 & 0,0524 & 0,1067 & 0,2207 & 0,4143 & 0,5052 & 0,6168 & 0,7555 \\
\hline
\end{tabular}

De acordo com Fioreze (1986), o déficit de pressão de vapor (DPV) relaciona a umidade de equilíbrio estático com a umidade de equilíbrio dinâmico, através das seguintes expressões:

$$
\begin{gathered}
\Delta \mathrm{U}=\text { Xeq dinâmico }- \text { Xeq estático } \\
\Delta \mathrm{U}=0,295 * \operatorname{Ln}[\mathrm{DPV}] \\
\mathrm{DVP}=(\mathrm{Pvs}-\mathrm{Pv})
\end{gathered}
$$

donde:

$\mathrm{Pv}$ - Pressão de vapor de água no alimento $(\mathrm{kPa})$

Tabela 1. Sais utilizados para o preparo das soluções saturadas e correspondentes atividades de água $\left(a_{w}\right)$ às temperaturas de 50 e 73 oC (Greenspan, 1977) 
Pvs - Pressão de vapor de água à saturação $(\mathrm{kPa})$

O melhor modelo obtido da etapa anterior foi utilizado para cálculo das umidades de equilíbrio estático (Xeq estático). Os dados utilizados no modelo de isoterma de sorção e na equação de Fioreze (1986) foram obtidos de testes de secagem realizados nas condições mostradas na Tabela 2.

Tabela 2. Condições do ar de secagem das amostras

\begin{tabular}{cccccccc}
\hline Amostras & $\begin{array}{c}\mathrm{Tbs}\left({ }^{\circ} \mathbf{C}\right) \\
\text { ambiente }\end{array}$ & $\begin{array}{c}\mathrm{Tbu}\left({ }^{\circ} \mathbf{C}\right) \\
\text { ambiente }\end{array}$ & $\begin{array}{c}\mathrm{Tbs}\left({ }^{\circ} \mathrm{C}\right) \\
\text { câmara }\end{array}$ & $\begin{array}{c}\mathrm{Tbu}\left({ }^{\circ} \mathbf{C}\right) \\
\text { câmara }\end{array}$ & $\begin{array}{c}\mathrm{Tbs}\left({ }^{\circ} \mathrm{C}\right) \\
\text { saída }\end{array}$ & $\begin{array}{c}\text { Tbu }\left({ }^{\circ} \mathbf{C}\right) \\
\text { saída }\end{array}$ & $\begin{array}{c}\text { Velocidade } \\
\left(\mathbf{m ~ s}^{-1}\right)\end{array}$ \\
A2 & 27,52 & 19,81 & 56,01 & 26,60 & 46,84 & 25,54 & 0,21 \\
A3 & 24,76 & 19,70 & 55,69 & 26,65 & 46,80 & 25,47 & 0,21 \\
A4 & 27,14 & 20,95 & 56,44 & 30,33 & 46,87 & 25,65 & 0,20 \\
A5 & 26,69 & 19,89 & 65,17 & 30,58 & 54,77 & 27,09 & 0,22 \\
A6 & 27,90 & 20,29 & 65,89 & 31,45 & 54,03 & 27,18 & 0,22 \\
A7 & 28,25 & 20,23 & 65,90 & 31,46 & 54,13 & 27,13 & 0,22 \\
A8 & 27,26 & 21,32 & 75,26 & 31,36 & 65,40 & 30,68 & 0,23 \\
A9 & 28,42 & 20,47 & 76,30 & 32,15 & 64,48 & 29,87 & 0,23 \\
A10 & 28,72 & 21,78 & 75,59 & 32,79 & 64,23 & 30,58 & 0,23 \\
A11* & 24,58 & 19,58 & 56,00 & 26,52 & 46,64 & 25,28 & 0,21 \\
A12* & 27,43 & 19,99 & 78,10 & 31,94 & 66,04 & 29,92 & 0,23 \\
A13* & 29,28 & 20,28 & 75,30 & 33,80 & 63,58 & 28,98 & 0,23 \\
\hline
\end{tabular}

Para cada condição de secagem (temperaturas de bulbo úmido e bulbo seco) do ar de exaustão e pressão barométrica de Campinas $(713,3 \mathrm{mmHg}$ ) com ajuda do programa para cálculo de propriedades e processos psicrométricos, elaborado por Biagi (2006), calcularam-se as pressões de vapor (Pv) e pressões de vapor à saturação (Pvs) usados na estimativa do déficit de pressão de vapor [DPV]. Com este dado se calculou $\Delta \mathrm{U}$ com as equações (15) e (16) e, posteriormente, o valor da umidade de equilíbrio dinâmico para cada condição de secagem.

\section{RESULTADOS E DISCUSSÃO}

O conteúdo de umidade inicial das amostras com $16,1 \%$ de mucilagem residual foi de 0,1047 b.s ( $\mathrm{cv}=1,58 \%)$, ao passo que nas amostras com 52,7\% de mucilagem residual, foi de $0,0847 \mathrm{bs}(\mathrm{cv}=0,65 \%)$.

Ao se analisarem os valores médios de umidade de equilíbrio correspondentes às atividades de água da Tabela 1, desconsideraram-se os dados resultantes das soluções dos sais $\mathrm{NaOH}$ e KOH, visto que apresentaram grande instabilidade; após essas considerações, os valores médios de umidade de equilíbrio com diferentes teores de mucilagem residual de acordo com a $\mathrm{a}_{\mathrm{w}}$ correspondente são apresentados na Tabela 3.

As isotermas de sorção do café cereja descascado (CCD) apresentaram formato sigmoidal típico de alimentos e materiais biológicos, podendo-se observar um pronunciado aumento da umidade de equilíbrio, a partir de atividades de água na faixa de 0,6 (Figura 1). Este comportamento é comum em alimentos com altos teores de açúcar, que têm a propriedade de absorver pequenas ou grandes quantidades de água, de acordo com a umidade relativa do ambiente, isto é, se baixa ou alta (Samaniego-Esguerra et al., 1991).
Tabela 3. Umidades de equilíbrio de CCD com teores de mucilagem residual de $16,1 \%$ (M1) e $52,7 \%$ (M2) nas temperaturas de 50 e $73^{\circ} \mathrm{C}$

\begin{tabular}{|c|c|c|c|c|c|c|}
\hline \multirow{3}{*}{ Sal } & \multicolumn{6}{|c|}{ XE $\left(\mathrm{kg}_{\mathrm{H} 20} \mathrm{~kg}_{\mathrm{ms}}^{-1}\right)$} \\
\hline & \multicolumn{3}{|c|}{$\mathrm{T}=50^{\circ} \mathrm{C}$} & \multicolumn{3}{|c|}{$\mathrm{T}=73^{\circ} \mathrm{C}$} \\
\hline & aw & M1 & M2 & aw & M1 & M2 \\
\hline $\mathrm{LiCl}$ & 0,1110 & 0,0349 & 0,0366 & 0,1067 & 0,0218 & 0,0215 \\
\hline $\mathrm{KF}$ & 0,2080 & 0,0655 & 0,0672 & 0,2207 & 0,0436 & 0,0445 \\
\hline $\mathrm{K}_{2} \mathrm{CO}_{3}$ & 0,4270 & 0,0699 & 0,0707 & 0,4143 & 0,0448 & 0,0453 \\
\hline $\mathrm{NaBr}$ & 0,5093 & 0,0814 & 0,0793 & 0,5052 & 0,0677 & 0,0659 \\
\hline $\mathrm{KI}$ & 0,6449 & 0,1004 & 0,0940 & 0,6168 & 0,0803 & 0,0799 \\
\hline $\mathrm{NaCl}$ & 0,7443 & 0,1275 & 0,1356 & 0,7555 & 0,1169 & 0,1131 \\
\hline
\end{tabular}

Nota-se, quanto ao efeito da temperatura, que para um mesmo valor de atividade de água quanto maior a temperatura menor a umidade de equilíbrio. Este resultado concorda com o reportado por Labuza et al. (1985). A partir dos parâmetros de ajuste estimados para os modelos matemáticos, traçaramse as isotermas preditas, juntamente com os dados experimentais para os dois modelos que apresentaram melhor ajuste (Tabela 4, Figura 1).

Os gráficos de distribuição de resíduos correspondentes ao ajuste dos modelos de Halsey Modificado e Peleg aos dados experimentais para 16,1 e 52,7\% de mucilagem e temperaturas de 50 e $73{ }^{\circ} \mathrm{C}$ mostram distribuição aleatória dos resíduos revelando ausência de tendência (Figura 2).

Na temperatura de $50{ }^{\circ} \mathrm{C}$, os modelos Peleg, Halsey Modificado, GAB e Oswin são os que melhor representam as isotermas de sorção do CCD com baixos teores de mucilagem $(16,1 \%)$, enquanto para $73{ }^{\circ} \mathrm{C}$, somente os modelos Peleg e Halsey Modificado conseguiram representar bem os dados experimentais, especialmente na região de baixa atividade de água, considerada de maior interesse no presente trabalho (Tabela 4).

O modelo de Halsey mostra desvios relativos médios comparáveis ao modelo de Halsey Modificado, mas em virtude dos graus de liberdade estatística do modelo, o desvio padrão da estimativa resultou em valores bem mais altos. O modelo GAB indica desvios padrão da estimativa comparáveis ao modelo de Peleg, mas seus DRM resultaram muito altos. O modelo BET linear, apesar de ter resultado em valores de DRM superiores a 10\%, mostrou boa representação dos dados experimentais.

A qualidade do ajuste para todos os modelos referentes à temperatura de $73{ }^{\circ} \mathrm{C}$ é bem melhor, tanto nos coeficientes de correlação quanto no desvio padrão da estimativa que para $50{ }^{\circ} \mathrm{C}$ (Tabela 3 ).

Figueira et al. (2004) encontraram, na avaliação de isotermas de adsorção da raiz de chicória (Chicorium intybus L.), da qual se faz uma bebida denominada 'café-chicória', que os dados experimentais às temperaturas de 60,70 e $80{ }^{\circ} \mathrm{C}$ foram melhor representados pelos modelos GAB e Peleg. Aviara et al. (2004) reportaram o modelo de Halsey Modificado como o melhor para a previsão da atividade de água em grãos de soja.

$\mathrm{O}$ modelo de Peleg também resultou em melhor ajuste em estudos de processo de adsorção de sementes da espécie arbórea Cedrela fissilis (Espindola, 2007) e de sementes de feijoeiro cultivar Uma (Francisco et al., 2007), na temperatura de $25^{\circ} \mathrm{C}$. 

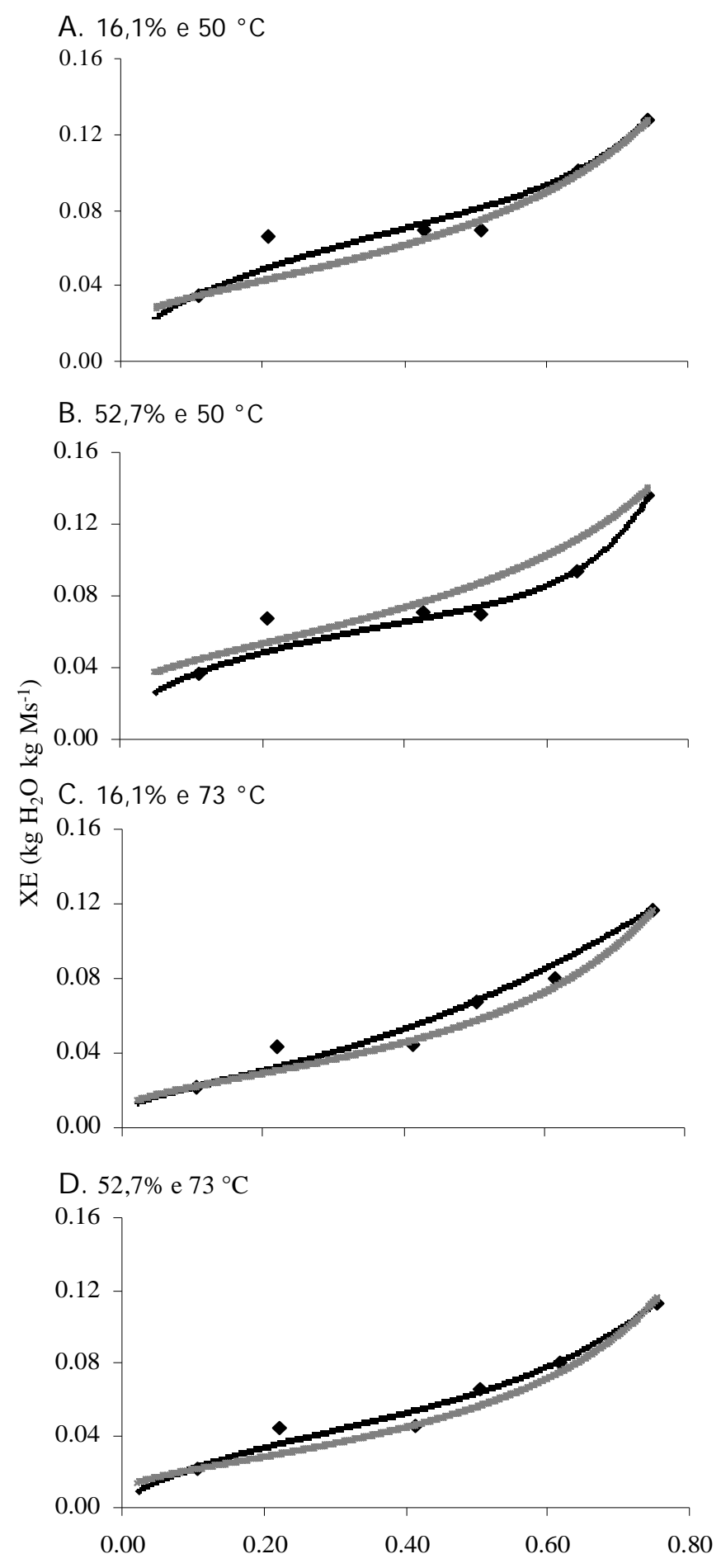

Peleg Halsey Modificado

- Dados experimentais

Figura 1. Isotermas de sorção teóricas (modelos Halsey Modificado e Peleg) e dados experimentais para o CCD com 16,1 e52,7\% de mucilagem nas temperaturas de 50 e $73^{\circ} \mathrm{C}$

No presente trabalho foi escolhido o modelo de Peleg para o cálculo das umidades de equilíbrio estático e dinâmico, em razão de apresentar os melhores indicadores $\left(\mathrm{R}^{2}\right.$, DRM e SE) e a distribuição de resíduos aleatória.
Tabela 4. Parâmetros de ajuste dos modelos matemáticos de isotermas de sorção aos dados experimentais para o CCD com $16,1 \%$ (M1) e $52,7 \%$ (M2) de mucilagem em temperatura de 50 e $73 \stackrel{\circ}{C}$, valores de $R^{2}$, DRM e SE correspondentes

\begin{tabular}{|c|c|c|c|c|c|}
\hline \multirow{2}{*}{ Modelo } & \multirow{2}{*}{ Parâmetros } & \multicolumn{2}{|c|}{$50 \stackrel{\circ}{\circ}$} & \multicolumn{2}{|r|}{$73 \stackrel{\circ}{\circ}$} \\
\hline & & M1 & M2 & M1 & M2 \\
\hline \multirow{6}{*}{ BET } & Xm & 0,7603 & 2,0308 & 0,6125 & 2,0597 \\
\hline & $C$ & 0,8656 & 0,7214 & 0,4574 & 0,0692 \\
\hline & $\mathrm{n}$ & 0,3575 & 0,1471 & 0,6086 & 0,8923 \\
\hline & R2 & 0,7903 & 0,6818 & 0,8254 & 0,8845 \\
\hline & DRM & 13,15 & 13,21 & 12,42 & 16,61 \\
\hline & $\begin{array}{c}S E \\
\left(g_{\text {agua }} g_{m s}{ }^{-1}\right)\end{array}$ & 0,0231 & 0,0296 & 0,0222 & 0,0173 \\
\hline \multirow{5}{*}{ BET linear } & Xm & 0,0357 & 0,0348 & 0,0287 & 0,0291 \\
\hline & C & 10000 & 80,4995 & 100,3117 & 16,0886 \\
\hline & $\mathrm{R} 2$ & 0,8692 & 0,8607 & 0,9505 & 0,9309 \\
\hline & DRM & 11,29 & 10,08 & 14,15 & 9,89 \\
\hline & $\begin{array}{c}\mathrm{SE} \\
\left(\mathrm{g}_{\text {agua }} \mathrm{g}_{\mathrm{ms}}^{-1}\right)\end{array}$ & 0,0258 & 0,0277 & 0,0118 & 0,0189 \\
\hline \multirow{6}{*}{ GAB } & $\mathrm{xm}$ & 0,0434 & 0,0421 & 0,0313 & 0,0276 \\
\hline & $C$ & 1000,100 & 180,4990 & 1000,3052 & 100000,5 \\
\hline & K & 0,8867 & 0,9228 & 0,9707 & 1,0010 \\
\hline & $\mathrm{R} 2$ & 0,9291 & 0,8598 & 0,9581 & 0,9346 \\
\hline & DRM & 11,59 & 9,46 & 14,74 & 15,56 \\
\hline & $\begin{array}{c}\mathrm{SE} \\
\left(\mathrm{g}_{\text {agua }} \mathrm{g}_{\mathrm{ms}}{ }^{-1}\right)\end{array}$ & 0,0141 & 0,0197 & 0,0109 & 0,0130 \\
\hline \multirow{5}{*}{ Halsey } & A & 0,0081 & 0,0082 & 0,1662 & 0,0197 \\
\hline & $b$ & 1,7466 & 1,7379 & 1,3297 & 1,2283 \\
\hline & $\mathrm{R} 2$ & 0,8924 & 0,8523 & 0,9226 & 0,9320 \\
\hline & DRM & 8,40 & 10,26 & 10,37 & 9,74 \\
\hline & $\begin{array}{c}\mathrm{SE} \\
\left(\mathrm{g}_{\text {agua }} \mathrm{g}_{\mathrm{ms}}{ }^{-1}\right)\end{array}$ & 0,0234 & 0,0286 & 0,0145 & 0,01873 \\
\hline \multirow{6}{*}{$\begin{array}{c}\text { Halsey } \\
\text { Modificado }\end{array}$} & Xm & 0,0635 & 0,0328 & 0,0273 & 0,0441 \\
\hline & $C$ & 1,0000 & 2,6912 & 1,7447 & 0,9231 \\
\hline & K & 1,6877 & 1,5392 & 1,2578 & 1,2298 \\
\hline & $\mathrm{R} 2$ & 0,8923 & 0,8765 & 0,9478 & 0,9320 \\
\hline & DRM & 8,39 & 10,27 & 9,69 & 9,74 \\
\hline & $\begin{array}{c}\mathrm{SE} \\
\left(\mathrm{g}_{\text {ácua }} \mathrm{g}_{\mathrm{ms}}{ }^{-1}\right)\end{array}$ & 0,01657 & 0,0185 & 0,0121 & 0,0133 \\
\hline \multirow{5}{*}{ Langmuir } & $X m$ & 0,1645 & 0,1390 & 0,1833 & 0,1855 \\
\hline & $C$ & 2,4239 & 3,2191 & 1,2624 & 1,2254 \\
\hline & R2 & 0,7626 & 0,6522 & 0,9653 & 0,8231 \\
\hline & DRM & 13,79 & 13,90 & 12,97 & 12,98 \\
\hline & $\begin{array}{c}\mathrm{SE} \\
\left(\mathrm{g}_{\text {agua }} \mathrm{g}_{\mathrm{ms}}{ }^{-1}\right)\end{array}$ & 0,0348 & 0,0438 & 0,0333 & 0,0303 \\
\hline \multirow{5}{*}{ Oswin } & $\mathrm{A}$ & 0,0801 & 0,077 & 0,0646 & 0,0635 \\
\hline & B & 0,3999 & 0,3624 & 0,5126 & 0,5104 \\
\hline & $\mathrm{R} 2$ & 0,9002 & 0,8082 & 0,9653 & 0,9633 \\
\hline & DRM & 8,34 & 10,27 & 8,47 & 7,74 \\
\hline & $\begin{array}{c}\mathrm{SE} \\
\left(\mathrm{g}_{\text {aqua }} \mathrm{g}_{\mathrm{ms}}{ }^{-1}\right)\end{array}$ & 0,0225 & 0,0326 & 0,0140 & 0,0138 \\
\hline \multirow{7}{*}{ Peleg } & $\mathrm{K} 1$ & 0,2667 & 0,5029 & 0,1534 & 0,1502 \\
\hline & N1 & 7,3034 & 7,3443 & 2,6693 & 4,7617 \\
\hline & K2 & 0,1132 & 0,0878 & 0,0492 & 0,0876 \\
\hline & N2 & 0,5353 & 0,3668 & 0,3709 & 0,6206 \\
\hline & $\mathrm{R} 2$ & 0,9207 & 0,9290 & 0,9511 & 0,9677 \\
\hline & DRM & 7,43 & 6,77 & 8,14 & 7,56 \\
\hline & $\begin{array}{c}\mathrm{SE} \\
\left(\mathrm{g}_{\text {áaua }} \mathrm{g}_{\mathrm{ms}}{ }^{-1}\right)\end{array}$ & 0,0116 & 0,01143 & 0,0093 & 0,0075 \\
\hline
\end{tabular}

\section{Umidade de equilíbrio dinâmico}

Os dados de umidade de equilíbrio variam com o tipo de sólido e podem ser determinados, experimentalmente, por métodos estáticos ou dinâmicos (Barrozo et al., 2000). 
A. $16,1 \%$ e $50^{\circ} \mathrm{C}$

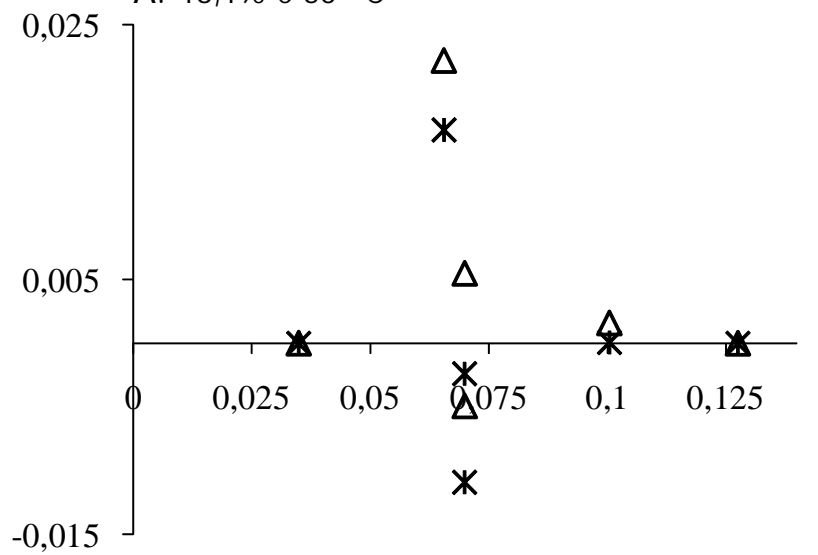

B. $52,7 \%$ e $50^{\circ} \mathrm{C}$

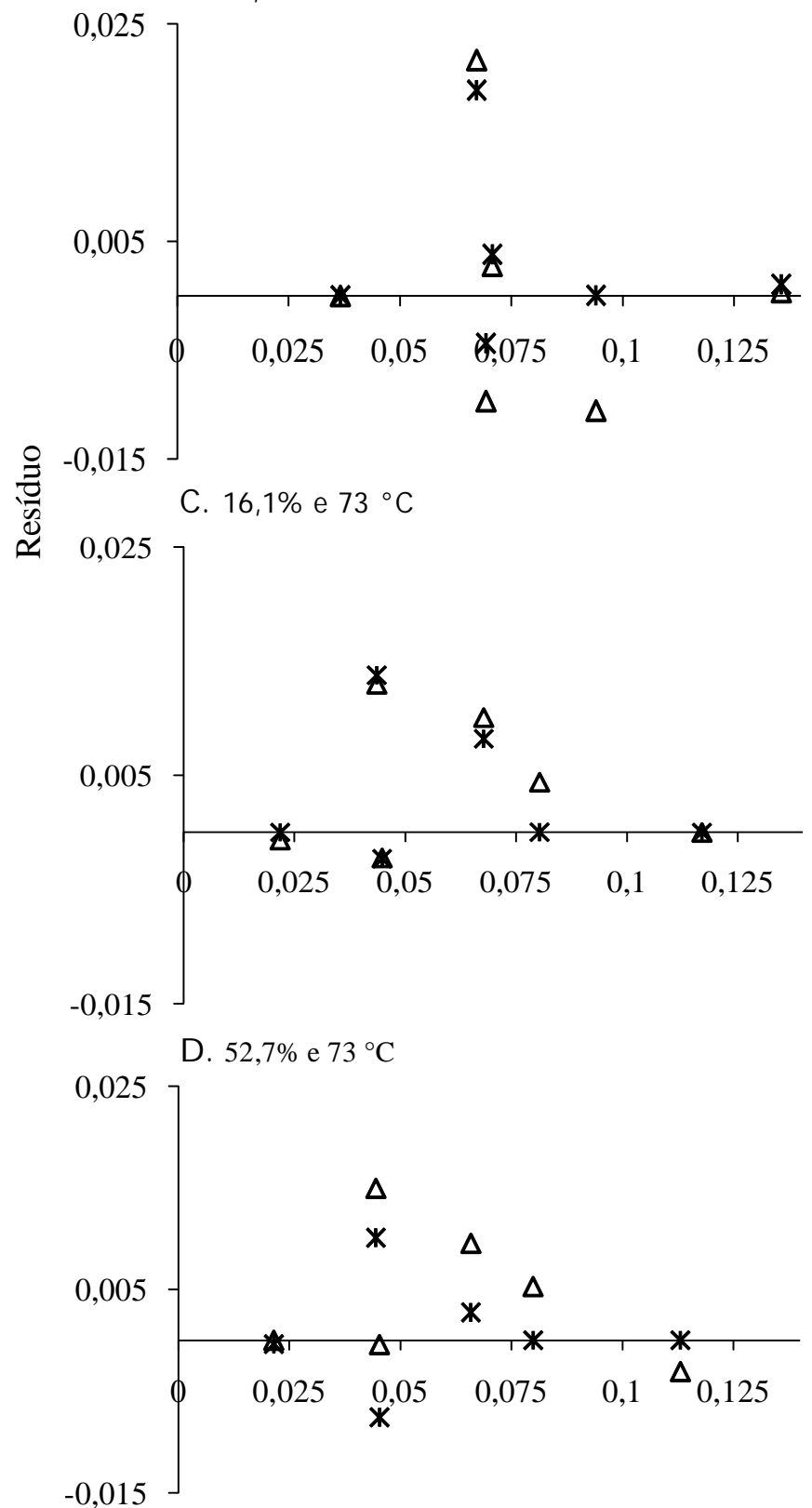

Umidade de equilíbrio (bs)

Figura 2. Distribuição dos resíduos dos modelos de $\mathrm{H}$ alsey Modificado $(\Delta)$ e Peleg $(\mathrm{x})$ para 16,1 e $52,7 \%$ de mucilagem nas temperaturas de 50 e $73 \stackrel{\circ}{\circ} \mathrm{C}$
A Tabela 5 apresenta as umidades de equilíbrio estático e dinâmico, juntamente com os parâmetros da equação proposta por Fioreze (1986). Com a finalidade de se avaliar a qualidade das expressões 14 a 16 para o cálculo da umidade de equilíbrio dinâmico, as amostras A4, A5, A8 e A12 foram secadas até atingir os valores experimentais de equilíbrio (Tabela 6).

Considerando-se a proximidade dos resultados experimentais (Tabela 6) com os correspondentes calculados (Tabela 5) é possível concluir que a equação proposta por Fioreze (1986) foi altamente satisfatória para o cálculo de umidade de equilíbrio dinâmico para o CCD.

Tabela 5. Umidades de equilíbrio das amostras de CCD com distintos teores de mucilagem submetidas a secagem

\begin{tabular}{|c|c|c|c|c|c|c|c|}
\hline Amostras & $\begin{array}{c}\text { Mucilagem } \\
(\%)\end{array}$ & UR(\%) & $\begin{array}{c}\text { Xeq } \\
\text { (est)(\% } \\
\text { b.s) }\end{array}$ & $\begin{array}{l}\text { PVS } \\
(\mathrm{kPa})\end{array}$ & $\mathrm{DPV}(\mathrm{kPa})$ & $\begin{array}{c}\Delta U \\
(\% \text { b.s. })\end{array}$ & $\begin{array}{c}\text { Xeq (din.) } \\
(\% \text { b.s })\end{array}$ \\
\hline$A 2$ & 63,5 & 18,86 & 4,72 & 10469,0 & 8494,547 & 1,81 & 6,53 \\
\hline A3 & 37,7 & 18,66 & 4,71 & 10469,0 & 8515,485 & 1,81 & 6,52 \\
\hline A4 & 22,4 & 18,99 & 4,75 & 10506,1 & 8510,992 & 1,81 & 6,56 \\
\hline A5 & 60,7 & 12,24 & 3,49 & 15492,4 & 13596,13 & 1,95 & 5,44 \\
\hline A6 & 46,4 & 13,15 & 3,65 & 14952,7 & 12986,42 & 1,93 & 5,58 \\
\hline A7 & 39,6 & 12,95 & 3,59 & 15024,5 & 13078,83 & 1,94 & 5,53 \\
\hline A8 & 57,6 & 9,12 & 2,45 & 25274,4 & 22969,37 & 2,10 & 4,55 \\
\hline A9 & 39,5 & 8,69 & 2,52 & 24260,7 & 22152,45 & 2,09 & 4,61 \\
\hline $\mathrm{A} 10$ & 29,3 & 9,97 & 2,46 & 23991,2 & 21599,28 & 2,08 & 4,54 \\
\hline A11* & 50,0 & 18,44 & 4,72 & 10384,5 & 8469,598 & 1,81 & 6,53 \\
\hline $\mathrm{A} 12^{*}$ & 52,7 & 7,81 & 2,22 & 26000,5 & 23969,86 & 2,11 & 4,33 \\
\hline A13* & 16,1 & 8,14 & 2,34 & 23302,4 & 21405,58 & 2,08 & 4,46 \\
\hline
\end{tabular}

Tabela 6. Umidades de equilíbrio dinâmico, experimentais e preditas

\begin{tabular}{cccc}
\hline Amostras & $\mathbf{U}_{\text {final }}$ obtida (\%) VE & $\mathbf{U}_{\text {final }}$ calculada (\%) VP & DRM (\%) \\
\hline A4 & 6,64 & 6,56 & 1,20 \\
A5 & 5,51 & 5,44 & 1,27 \\
A8 & 4,91 & 4,55 & 2,30 \\
A12 & 4,32 & 4,33 & 0,23 \\
\hline
\end{tabular}

\section{CONCLUSÕES}

1. As isotermas de dessorção para a temperatura de $50{ }^{\circ} \mathrm{C}$ e teores de mucilagem residual de 16,1 e $52,7 \%$ podem ser representadas pelos modelos: Peleg, Halsey Modificado, GAB e Oswin, nesta ordem. Para a temperatura de $73{ }^{\circ} \mathrm{C}$, o modelo GAB não representa bem os dados experimentais, sendo que os modelos Peleg e Halsey Modificado apresentam o melhor desempenho.

2. Para a estimativa das umidades de equilíbrio dinâmicas a equação proposta por Fioreze mostrou-se altamente satisfatória.

\section{AGRADECIMENTOS}

Agradecemos à Universidad Surcolombiana; ao Conselho Nacional de Desenvolvimento Científico e Tecnológico (CNPq); à Fundação de Amparo à Pesquisa do Estado de São 
Paulo (FAPESP), e à Faculdade de Engenharia Agrícola (FEAGRI/UNICAMP).

\section{LITERATURA CITADA}

Aguerre, R. J.; Gabitto, J. F.; Chirife, J. Utilization of Fick's second law for the evaluation of diffusion coefficients in food process controlled by internal diffusion. Journal of Food Technology, v.20, n.5, p.623-629, 1985.

AOAC - Association of Official Analytical Chemists. Official methods of analysis of AOAC International. 16.ed., v.2. Arlington: AOAC, 1995. 559p.

Aviara, N. A.; Ajibola, O. O.; Oni, S. A. Sorption equilibrium and thermodinamic characteristics of soja bean. Biosystems Engineering, v.87, n.2, p.179-190, 2004.

Barrozo, M. A. S.; Oliveira, D. T., Sancineti, G. P., Rodrigues, M. V. A study of the desorption isotherms of lentils. Brazilian Journal of Chemical Engineering, v.17, p.105-109, 2000.

Biagi, J. D. Programa para cálculo de propriedades e processos psicrométricos. Campinas: FEAGRI/UNICAMP, 2006.

Daudin, J. D. Calcul des cinétiques de séchage par l'air chaud des produits biologuiques solides. Sciences des Aliments, v.3, p.1-36, 1983.

Espindola, L. F. Determinação do teor de água ideal para o armazenamento de sementes de quatro espécies arbóreas ameaçadas da flora brasileira. Rio de Janeiro: Instituto de Pesquisas Jardim Botânico do Rio de Janeiro/Escola Nacional de Botânica Tropical, 2007. 61p. Dissertação Mestrado

Figueira, G. M.; Park, K. J.; Brod, F. P. R.; Honório, S. L. Evaluation of desortion isotherms, drying rates and inulin concentration of chicory roots (Chichorium intybus $\mathrm{L}$ ) with and without enzymatic inactivation. Journal of Food Engineering, v.63, n.3, p.273-280, 2004.
Fioreze, R. The intermittent drying of agricultural crops with particular reference to energy requirements. Bedford: Cranfield Institute of Technology Silsoe College. 1986. 153p. Ph D. Thesis

Fortes, M.; Okos, M. R. Heat and mass transfer in hygroscopic capillary extruded products. In: Annual Meeting of the AICHE, 71, 1978, Miami. Proceedings... Miami: Aiche, 1978.

Francisco, F. G.; Usberti, R.; Toneli, J. T. C. L. Ajuste de isotermas de sorção de sementes de cultivares de feijoeiro. Revista Brasileira de Sementes; v.29, n.1, p.35-39, 2007.

Greenspan, L. Humidity fixed points of binary saturated aqueous solutions. Journal of Research of the National Bureau of Standards - A. Physics and Chemistry, v.81A, n.1, p.89-96, 1977.

Labuza, T. P.; Kaanane, A.; Chen, J. Y. Effect of temperature on the moisture sorption isotherms and water activity shift of two dehydrated foods. Journal of Food Science, v.50, n.2, p.385-391, 1985.

Lomauro, C. J.; Bakshi, A. S.; Labuza, T. P. Moisture transfer properties of dry and semimoist foods. Journal of Food Science, v.50, n.2, p.397-400, 1985.

Salwin, H.; Slawson, V. Moisture transfer in combinations of dehydrated foods. Food Technology, v.13, n.12, p.715-718, 1959.

Samaniego-Esguerra, C. M.; Boag, I. F.; Robertson, G. L. Comparison of regression methods for fitting the GAB model to the moisture isotherms of some dried fruit and vegetables. Journal of Food Engineering, v.13, n.2, p.115133, 1991.

Scott, W. J. Water relations of the food spoilage microorganisms. Advances in Food Research, v.7, n.1, p.83-127, 1957.

Toneli, J. T. C. L. Processo de separação física de inulina obtida a partir de raízes de chicória (Chichorium intibus L.) Campinas: UNICAMP, 2006. 177p. Tese Doutorado 